\title{
Predict and Match: Prophet Inequalities with Uncertain Supply
}

\author{
Reza Alijani \\ alijani@cs.duke.edu \\ Duke University
}

\author{
Siddhartha Banerjee \\ sbanerjee@cornell.edu \\ Cornell University
}

\author{
Sreenivas Gollapudi \\ sgollapu@google.com \\ Google Research
}

\author{
Kamesh Munagala \\ kamesh@cs.duke.edu \\ Duke University
}

\author{
Kangning Wang \\ knwang@cs.duke.edu \\ Duke University
}

\begin{abstract}
We consider the problem of selling perishable items to a stream of buyers in order to maximize social welfare. A seller starts with a set of identical items, and each arriving buyer wants any one item, and has a valuation drawn i.i.d. from a known distribution. Each item, however, disappears after an a priori unknown amount of time that we term the horizon for that item. The seller knows the (possibly different) distribution of the horizon for each item, but not its realization till the item actually disappears. As with the classic prophet inequalities, the goal is to design an online pricing scheme that competes with the prophet that knows the horizon and extracts full social surplus (or welfare).

Our main results are for the setting where items have independent horizon distributions satisfying the monotone-hazard-rate (MHR) condition. Here, for any number of items, we achieve a constant-competitive bound via a conceptually simple policy that balances the rate at which buyers are accepted with the rate at which items are removed from the system. We implement this policy via a novel technique of matching via probabilistically simulating departures of the items at future times. Moreover, for a single item and MHR horizon distribution with mean $\mu$, we show a tight result: There is a fixed pricing scheme that has competitive ratio at most $2-1 / \mu$, and this is the best achievable in this class.

We further show that our results are best possible. First, we show that the competitive ratio is unbounded without the MHR assumption even for one item. Further, even when the horizon distributions are i.i.d. MHR and the number of items becomes large, the competitive ratio of any policy is lower bounded by a constant greater than 1 , which is in sharp contrast to the setting with identical deterministic horizons.
\end{abstract}

\section{ACM Reference Format:}

Reza Alijani, Siddhartha Banerjee, Sreenivas Gollapudi, Kamesh Munagala, and Kangning Wang . 2020. Predict and Match: Prophet Inequalities with Uncertain Supply. In ACM SIGMETRICS / International Conference on Measurement and Modeling of Computer Systems (SIGMETRICS '20 Abstracts), fune 8-12, 2020, Boston, MA, USA. ACM, New York, NY, USA, 2 pages. https://doi.org/10.1145/3393691.3394212

Permission to make digital or hard copies of part or all of this work for personal or classroom use is granted without fee provided that copies are not made or distributed for profit or commercial advantage and that copies bear this notice and the full citation on the first page. Copyrights for third-party components of this work must be honored. For all other uses, contact the owner/author(s).

SIGMETRICS '20 Abstracts, fune 8-12, 2020, Boston, MA, USA

(c) 2020 Copyright held by the owner/author(s).

ACM ISBN 978-1-4503-7985-4/20/06.

https://doi.org/10.1145/3393691.3394212

\section{INTRODUCTION}

Online posted pricing problems are one of the canonical examples in online decision-making and optimal control. The basic model comprises of a fixed supply of non-replenishable items; buyers (demand) arrive in an online fashion over a fixed time interval, and the platform sets prices to maximize some objective such as social surplus (welfare) or revenue. Another variant of this setting is found in internet advertising, where the number of advertisements (supply) is assumed to be fixed (for example, based on contracts between the publisher and advertisers), while keywords/impressions (demand) arrive online, and are matched to ads via some policy. The demand is typically assumed to obey some underlying random process, which allows the problem to be cast as a Markov Decision Process (MDP); however, in many settings, such a formulation suffers from a "curse of dimensionality", making it infeasible to solve optimally.

An important idea for circumventing the computational intractability of optimal pricing is that of prophet inequalities - heuristics with performance guarantees with respect to the optimal policy in hindsight (i.e., the performance of a prophet with full information of future arrivals).

The aim of our work is to develop a theory of prophet inequalities for settings with uncertainty in future supply which is a natural extension of the basic posted-price setting. What makes these problems of greater relevance today is the rise of online 'sharing economy' marketplaces. The novelty in such marketplaces arises because of their two-sided nature: in addition to buyers who arrive online, the supply is now controlled by "sellers" who can arrive and depart in an online fashion. For example, in the case of ridesharing/lodging platforms, the units of supply (empty vehicles/vacant listings) arrive over time, and have some patience interval after which they abandon the system (get matched to rides on other platforms/remove their listings). Supply uncertainty also arises in other settings, for instance, if items are perishable and last for $a$ priori random amounts of time. Our work aims to understand the design of pricing policies for such settings, and characterize how the resulting prophet inequalities depend on the characteristics of the supply uncertainty.

\subsection{Model}

We introduce "supply uncertainty" into the basic prophet inequality setting as follows: There are $m$ items present initially, but these do not last till the end of the buyer arrivals, but instead, depart after an a priori unknown amount of time. Formally, we assume each item $i$ samples a horizon from a distribution $H_{i}$, at which it departs. We assume the horizon lengths for items are mutually independent, 
and also independent of the valuation distribution of the buyers. Note though that the items can have different horizon distributions. We denote the maximum possible horizon length for any item as $n$.

On the demand side, we assume there is an infinite stream of unit-demand buyers arriving online, where the valuation of the $h$-th arriving buyer is a random variable $X_{h}$ drawn i.i.d. from a distribution $V$. From the perspective of a buyer, all items are interchangeable, and hence being matched to any item that has not yet departed yields value $X_{h}$. Note that assuming an infinite stream of buyers is without loss of generality, because we can encode any upper bound on the number of buyers in the horizon distributions.

The algorithm designer knows the horizon distribution $H_{i}$ for each item, and the buyer value distribution $V$, but not the realized horizons for each item (until the item actually departs), or the value for any buyer. The goal is to design an online pricing scheme that competes with a prophet that knows the realized horizons of each item and the valuation sequence of buyers, and extracts full social surplus (or welfare).

The main outcome of the standard prophet inequality is that there are constant-competitive algorithms for maximizing welfare, even when buyers are heterogeneous and arrive in arbitrary order. This however turns out to be impossible in the presence of item horizons without additional assumptions. Even with i.i.d. horizons, achieving a constant factor turns out to be impossible for general horizon distributions ( $c f$. Theorem 1.5); thus to make progress, we need more structure on the horizons. One natural assumption is that each item is more and more likely to depart as time goes on, which can be formalized as follows.

Definition 1.1. A horizon distribution $H$ satisfies the monotonehazard-rate (MHR) condition if:

$\operatorname{Pr}_{h \sim H}\left[h \geq h^{*}+2 \mid h \geq h^{*}+1\right] \leq \operatorname{Pr}_{h \sim H}\left[h \geq h^{*}+1 \mid h \geq h^{*}\right], \quad \forall h^{*} \geq 1$.

Several distributions satisfy the MHR condition, including uniform, geometric, deterministic, and Poisson; note also that truncating an MHR distribution preserves the condition.

\subsection{Main Result: Prophet Inequalities under Uncertain Supply}

The above discussion motivates us to study settings with MHR horizons. Our main result is that this assumptions is sufficient to obtain a constant-competitive approximation to the prophet welfare. In particular, our main technical result is the following theorem.

THEOREM 1.2. There is a constant-competitive online policy for social surplus for any $m \geq 1$ items with independent and possibly non-identical MHR horizon distributions, and unit-demand buyers arriving with i.i.d. valuations.

Though the complete algorithm is somewhat involved, at a high level, it is based on a simple underlying idea: to be constantcompetitive against the prophet, we need to choose prices so as to balance the rate of matches and departures. Achieving this in the general case is non-trivial, and requires some new technical ideas. However, for the special case of a single item, balancing can be achieved via a simple fixed pricing scheme. We use this to obtain the following tight result for the $m=1$ setting:
THEOREM 1.3. There is a fixed pricing scheme for a single item with an MHR horizon distribution with mean $\mu$ that has competitive ratio $2-1 / \mu$. Further, this bound is tight for the geometric horizon distribution with mean $\mu$.

Intuitively, the factor of two in the above theorem corresponds to the prophet considering matching and departures as the same, which an algorithm cannot do. The surprising aspect is that this simple policy is worst-case optimal within the class of instances with MHR horizons - this is in contrast to deterministic horizons, where fixed pricing is known to be suboptimal for the special case of one item with known (deterministic) horizon and i.i.d. buyers.

\subsection{Lower Bounds}

We complement our positive results by showing several lower bounds that establish their tightness. As mentioned above, we show a (tight) lower bound of $2-1 / \mu$ for $m=1$ items with MHR horizons. Our main lower bounds generalize this to $m \geq 1$ items.

THEOREM 1.4. For the multi-item setting with i.i.d. geometric horizons:

- For any number of items, there is a lower bound of 1.57 on the competitive ratio of any dynamic pricing scheme; in the limit when the number of items goes to infinity, this improves to 2.

- No fixed pricing scheme can be o( $\log \log m)$-competitive where $m$ is the number of items.

The above theorem implies that the MHR horizon setting, even with i.i.d. horizons, is significantly different from the setting with multiple items and a single deterministic horizon (where fixed pricing extracts $\left(1-O\left(\frac{1}{\sqrt{m}}\right)\right)$-fraction of surplus). Put differently, the lower bound emphasizes that even with i.i.d. horizons, to obtain a constant-competitive algorithm, it is not sufficient to replace the horizon distributions by their expectations and use standard prophet inequalities - the stochastic nature of the horizons allows for significant deviations in the order of departures of the items, and a policy that knows this ordering can potentially extract much more welfare. Given this, it is quite surprising that a simple dynamic pricing scheme achieves a constant approximation.

Finally, we consider the general case where there is no restriction on the horizon distribution. In this setting, the presence of supply uncertainty severely limits the performance of any non-anticipatory dynamic pricing scheme in comparison to the omniscient prophet. In particular, we show that for any number of items and i.i.d. buyer valuations, the ratio between the welfare of any algorithm and the prophet grows with the horizon, even if the algorithm knows the realized valuations.

THeorem 1.5. For any $m \geq 1$ items, there exists a family of instances such that the prophet has welfare $\Omega\left(\frac{\log n}{\log \log n}\right)$-factor larger than any online policy, even if the policy knows all the realized values, but not the realized horizons. Here, $n=\max _{i}\left\{\operatorname{supp}\left(H_{i}\right)\right\}$.

\section{ACKNOWLEDGMENTS}

This work is supported by NSF grants ECCS-1847393, DMS-1839346, CCF-1408784, CCF-1637397, and IIS-1447554; ARL award W911NF17-1-0094; ONR award N00014-19-1-2268; and research awards from Adobe and Facebook. 